\title{
openheart Discrepancy between left ventricular hypertrophy by echocardiography and electrocardiographic hypertrophy: clinical characteristics and outcomes
}

\author{
Yuta Seko, ${ }^{1}$ Takao Kato (D) , ${ }^{1}$ Yuhei Yamaji, ${ }^{2}$ Yoshisumi Haruna, ${ }^{2}$ Eisaku Nakane,${ }^{2}$ \\ Tetsuya Haruna, ${ }^{2}$ Moriaki Inoko ${ }^{2}$
}

- Additional supplemental material is published online only. To view, please visit the journal online (http://dx.doi.org/10. 1136/openhrt-2021-001765).

To cite: Seko Y, Kato T, Yamaji Y, et al. Discrepancy between left ventricular hypertrophy by echocardiography and electrocardiographic hypertrophy: clinical characteristics and outcomes. Open Heart 2021;8:e001765. doi:10.1136/ openhrt-2021-001765

Received 23 June 2021 Accepted 7 September 2021

Check for updates

(C) Author(s) (or their employer(s)) 2021. Re-use permitted under CC BY-NC. No commercial re-use. See rights and permissions. Published by BMJ.

${ }^{1}$ Department of Cardiovascular Medicine, Kyoto University Graduate School of Medicine, Kyoto, Japan

${ }^{2}$ Cardiovascular Center, Tazuke Kofukai Medical Research Institute, Kitano Hospital, Osaka, Japan

Correspondence to Dr Takao Kato; tkato75@kuhp. kyoto-u.ac.jp

\section{ABSTRACT}

Background The clinical significance of the discrepancy between left ventricular hypertrophy (LVH) by echocardiography and ECG remains to be elucidated. Methods After excluding patients who presented with pacemaker placement, QRS duration $\geq 120 \mathrm{~ms}$ and cardiomyopathy and moderate to severe valvular disease, we retrospectively analysed 3212 patients who had undergone both scheduled transthoracic echocardiography (echo) and ECG in a hospital-based population. Cornell product $>2440 \mathrm{~mm} \cdot \mathrm{ms}$ was defined as ECG-based LVH; left ventricular mass index $>115 \mathrm{~g} / \mathrm{m}^{2}$ for men and $>95 \mathrm{~g} /$ $\mathrm{m}^{2}$ for women was defined as echo-based LVH. The study population was categorised into four groups: patients with both ECG-based and echo-based LVH ( $\mathrm{N}=131,4.1 \%)$, those with only echo-based LVH $(\mathrm{N}=156,4.9 \%)$, those with only ECG-based LVH ( $\mathrm{N}=409,12.7 \%)$ and those with no LVH (N=2516, 78.3\%).

Results The cumulative 3-year incidences of a composite of all-cause death and major adverse cardiovascular events were $32.0 \%, 33.8 \%, 19.2 \%$ and $15.7 \%$, respectively. After adjusting for confounders, the HRs relative to that in no $\mathrm{LVH}$ were $1.63(95 \% \mathrm{Cl} 1.16$ to 2.28$)$, 1.68 (95\% Cl 1.23 to 2.30$)$ and 1.09 (95\% Cl 0.85 to 1.41$)$ in patients with both ECG-based and echo-based LVH, those with only echo-based LVH, and those with only ECGbased LVH, respectively.

Conclusions Echo-based LVH without ECG-based LVH was associated with a significant risk of adverse clinical events, and the risk was comparable to that in patients with both echo-based and ECG-based LVH.

\section{INTRODUCTION}

ECG and echocardiography (echo) are common and painless non-invasive methods for detecting several heart problems. ECG provides unique information on the electrical activity of the heart, while an echo provides structural and functional information in healthy and diseased individuals.

Left ventricular hypertrophy (LVH) is the hallmark of pressure or volume overload or structural change irrespective of overload,

\section{Key questions}

What is already known about this subject?

- When left ventricular hypertrophy (LVH) is evaluated simultaneously by ECG and echo, discrepancies between ECG-based LVH and echo-based LVH are observed in a certain proportion of patients.

What does this study add?

Echo-based LVH without ECG-based LVH was associated with a significant risk of adverse clinical events, and the risk was comparable to that in patients with both echo-based and ECG-based LVH.

How might this impact on clinical practice?

- When we see the mismatch between ECG-based LVH and echo-based LVH, it is necessary to thoroughly investigate the underlying mechanism that causes the mismatch in order to understand and treat the pathophysiological condition in each patient.

such as hypertrophic cardiomyopathy. Many previous studies have evaluated the prognostic impact of ECG-based $\mathrm{LVH}^{12}$ or echo-based LVH. ${ }^{34} \mathrm{LV}$ structure does not always relate to electrical activity in the myocardium and vice versa. For example, echo-based LVH is often observed in patients with cardiac amyloidosis, but the voltage of electrical activity is not large. The increased voltage is implicitly considered as a function of the $\mathrm{LV}$, which is in agreement with the echocardiographic findings. ECG-based LVH patterns are also seen because the slowing of the conduction velocity changes the sequence of ventricular activation even in situations where the anatomy of the left ventricle is not changed. It has been shown that slowed conduction due to fibrosis could also lead to an increase in QRS voltage, not necessarily associated with increased LV mass. ${ }^{5}$ In fact, when LVH is evaluated simultaneously by ECG and echo, discrepancies between ECG-based LVH and echo-based LVH are observed in a certain 


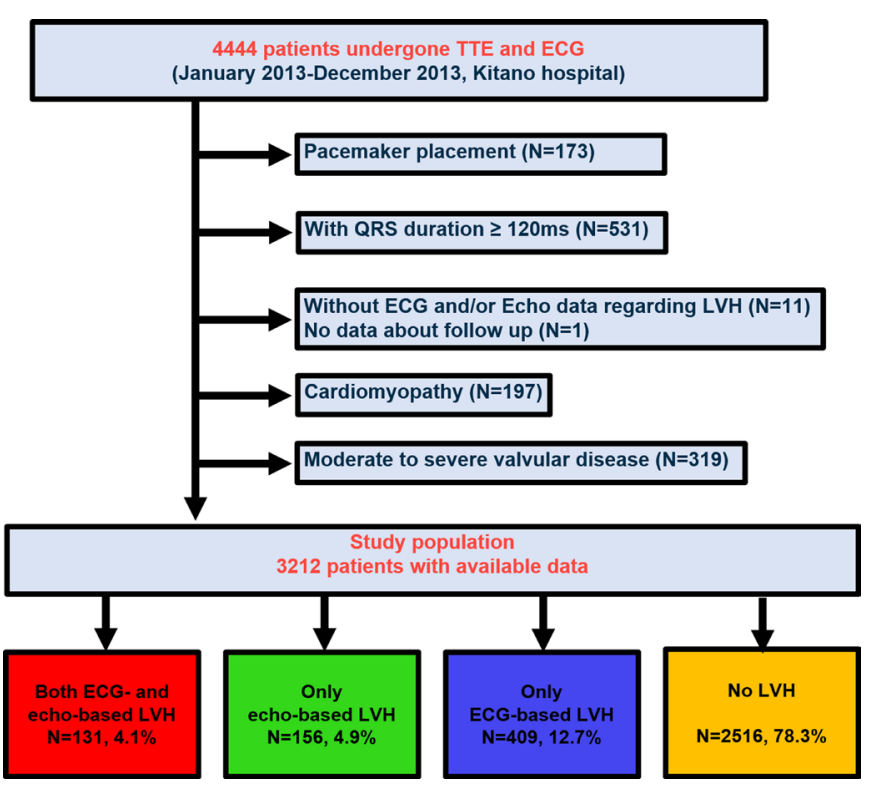

Figure 1 Patient flowchart. LVH, left ventricular hypertrophy; TTE, transthoracic echocardiography.

proportion of patients. However, there is a scarcity of data regarding the prognostic implication of the discrepant pattern, such as with echo-based LVH without ECG-based LVH and with ECG-based LVH without echo-based LVH. ${ }^{6}$ Thus, we aimed to characterise these patients and test the hypothesis that patients with discrepant patterns, such as echo-based LVH without ECG-based LVH and ECGbased LVH without echo-based LVH have worse clinical outcomes than those without LVH in a hospital-based population in Japan.

\section{METHODS}

\section{Study design, setting and population}

We retrospectively analysed 4444 patients who had undergone simultaneous scheduled transthoracic echo and ECG at Kitano Hospital in 2013 at the physician's discretion. Both outpatients and inpatients were included in the study. A flowchart of the study population is shown in figure 1. We excluded 1232 patients with pacemaker placement $(\mathrm{N}=173)$, QRS duration $\geq 120 \mathrm{~ms}(\mathrm{~N}=531)$, no ECG and/or echo data regarding LVH $(\mathrm{N}=11)$, no data regarding follow-up $(\mathrm{N}=1)$, cardiomyopathy $(\mathrm{N}=197)$ and moderate to severe valvular disease (ie, aortic stenosis, aortic regurgitation, mitral stenosis, mitral regurgitation and tricuspid regurgitation, $\mathrm{N}=319$ ). The study population comprised 3212 patients, who were categorised into four groups: patients with both ECG-based and echobased LVH, those with only echo-based LVH, those with only ECG-based LVH and those with no LVH (those without ECG-based or echo-based LVH).

\section{Ethics}

The requirement for informed consent was waived by the institutional review board of Kitano Hospital because of the retrospective study design. ${ }^{78}$ We disclosed the details of the present study to the public as an opt-out method and clearly informed patients of their right to refuse enrolment. The study protocol conformed to the ethical guidelines of the 1975 Declaration of Helsinki, as reflected in a priori approval by the institution's Human Research Committee. Patient records and information were anonymised and deidentified prior to the analysis.

\section{Definitions of ECG-and echo-based LVH and data collection}

ECG was measured at a paper speed of $25 \mathrm{~mm} / \mathrm{s}$, at a gain of $10 \mathrm{~mm} / \mathrm{mV}$ (or $5 \mathrm{~mm} / \mathrm{mV}$ ), using ECG devices including QRS duration and axis, PR interval, QT interval and heart rate (ECG data management system EFS-8800, Fukuda Denshi, Tokyo). All ECG data were calculated using ECG computer software. QRS duration was measured automatically to the nearest $1 \mathrm{~ms}$ and QRS amplitude to the nearest $10 \mu \mathrm{V}$. Cornell voltage was measured as SV3 + RaVL. ${ }^{910}$ Cornell product was calculated as the product of QRS duration times Cornell voltage in men and the product of QRS duration times Cornell voltage plus $6 \mathrm{~mm}$ in women, and ECG criteria for LVH using the Cornell product were defined as $>2440 \mathrm{~mm} \cdot \mathrm{ms}^{11-13} \mathrm{We}$ performed the additional analysis using two additional LVH criteria. The criteria for LVH in Cornell voltage were defined as $>2.8 \mathrm{mV}(28 \mathrm{~mm})$ in men and $>2.0 \mathrm{mV}(20$ $\mathrm{mm}$ ) in women. ${ }^{9}$ Sokolow-Lyon voltage was measured as SV1 +RV5 or RV6, and criteria for LVH were defined as $\geq 3.5 \mathrm{mV}(35 \mathrm{~mm})$. $^{9}$

Using the transthoracic echo database, we extracted data regarding wall thickness, LV diastolic dimension, LV systolic dimension, left atrial dimension, left atrial volume index (LAVI), LV ejection fraction (LVEF), transmitral flow, tissue Doppler imaging, valvular status and body mass index (BMI). ${ }^{7} 8$ 14-18 Based on the transthoracic echo data along with the catheter suite database, we identified patients with previous myocardial infarction or structural heart disease. Echocardiographic LVH was reported in the final echo report via standard calculation from measurements made by a technician. LV mass index (LVMI) and relative wall thickness (RWT) were calculated using the formula recommended by the American Society of Echocardiography. ${ }^{19}$ Echocardiographic LVH was defined as a high LVMI: LVMI $>115 \mathrm{~g} / \mathrm{m}^{2}$ for men and $>95 \mathrm{~g} / \mathrm{m}^{2}$ for women. High RWT was defined as RWT $>0.42$. High LAVI was defined as LAVI>34 mL/ $\mathrm{m}^{2}$. LVEF was measured using the Teichholz or modified Simpson's rule methods. All transthoracic echo measurements were performed using an average of at least three cardiac cycles.

We also extracted patient information from the electronic medical records at our institution, including age, sex and type of disease (ie, ischaemic heart disease, International Statistical Classification of Diseases and Related Health Problems, Tenth Revision (ICD-10) codes I20, I21, I22, I23, I24 and I25; hypertension, ICD-10 codes I10, I11, I12, I13, I14 and I15; dyslipidaemia, ICD-10 code E78; diabetes mellitus, ICD-10 codes E10, E11, E12, E13 and E14 and chronic kidney disease, ICD-10 code N18). ${ }^{78}$ Follow-up data from serial clinic visits until June 
2017 were also retrospectively collected from the electronic medical records.

\section{Outcome measures}

The primary outcome was a composite of all-cause death or major adverse cardiovascular events (MACE), defined as acute heart failure, acute myocardial infarction, unstable angina pectoris, cerebral infarction, cerebral haemorrhage and emerging aorta and peripheral vascular disease, including treatment for aortic aneurysm, all of which required unplanned hospitalisation. The secondary outcomes were all-cause death and MACE, respectively. ${ }^{78}$

\section{Statistical analysis}

Categorical variables are presented as numbers with percentages and were compared using the $\chi^{2}$ test. Continuous variables are expressed as means with SD and were compared using one-way analysis of variance. We compared the patient characteristics and 3-year clinical outcomes among the four groups (no LVH (reference), those with only ECG-based LVH, those with only echo-based LVH and those with both ECG-based and echo-based LVH). The cumulative incidences of clinical events were estimated using the Kaplan-Meier method, and intergroup differences were assessed using log-rank tests. Multivariable Cox proportional hazards models were used to estimate the risk of primary and secondary outcomes associated with the four groups. The model also included the following 10 clinically relevant covariates: age as a continuous variable, sex, BMI $>25 \mathrm{~kg} / \mathrm{m}^{2}$, diabetes, hypertension, dyslipidaemia, ischaemic heart disease, chronic kidney disease, atrial fibrillation and LVEF $<50 \%$. The results were expressed as HRs and 95\% CIs. We also performed the subgroup analysis for the primary outcome measure stratified by ischaemic heart disease, chronic kidney disease, LAVI and RWT. We evaluated the interactions between the subgroup factors and the effects of LVH groups to the no LVH on the primary outcome measure. In the sensitivity analysis, we changed the variables BMI and LVEF as the continuous variable in the multivariable Cox proportional hazard models. In the additional analyses, we used two classifications regarding Cornell voltage and Sokolow-Lyon voltage for ECG-based LVH. All statistical analyses were performed by physicians (YS and TK) using JMP V.15 (SAS Institute, Cary, North Carolina, USA). All reported p values were two tailed, and the level of statistical significance was set at $\mathrm{p}<0.05$.

\section{RESULTS}

\section{Baseline clinical characteristics}

The study population was categorised into four groups: patients with both ECG-based and echo-based LVH $(\mathrm{N}=131,4.1 \%)$, those with only echo-based LVH $(\mathrm{N}=156$, $4.9 \%)$, those with only ECG-based LVH (N=409, $12.7 \%)$ and those without ECG-based or echo-based LVH (reference: $\mathrm{N}=2516,78.3 \%$ ) (figure 1). The baseline characteristics are presented in table 1. Patients with ECG-based and/or echo-based LVH were older and more likely to have diabetes, hypertension, dyslipidaemia, ischaemic heart disease and chronic kidney disease. On echocardiographic findings, those with ECG-based and/or echobased LVH had a high LVMI, high LAVI, a greater left LV dimension and RWT and a decreased LVEF (table 1); however, the magnitude was lower in those with only ECG-based LVH.

\section{Clinical outcomes}

The median follow-up duration was 1268 (IQR: 404-1456) days, with a $79.7 \%$ follow-up rate at 1 year. The cumulative 3 -year incidences of the primary outcome measure were $32.0 \%, 33.8 \%, 19.2 \%$ and $15.7 \%$, respectively (log-rank $\mathrm{p}<0.001$ ) (figure 2A). After adjusting for confounders, the excess risk of primary outcome measure remained significant in patients with both ECG-based and echobased LVH (HR 1.63; $95 \%$ CI 1.16 to 2.28; $\mathrm{p}=0.005$ ) and in those with only echo-based LVH (HR 1.68; 95\% CI 1.23 to $2.30 ; p=0.001$ ) relative to the no $\mathrm{LVH}$ group, while the excess risk of primary outcome measure was not significant in those with only ECG-based LVH (HR 1.09; 95 \% CI 0.85 to $1.41 ; \mathrm{p}=0.49$ ) (table 2 ). Although the cumulative 3 -year incidences of all-cause death and the adjusted risk were not different among the four groups (figure $2 \mathrm{~B}$ and table 2), the trend in MACE was fully consistent with the primary outcome measures (figure $2 \mathrm{C}$ and table 2 ). The description of MACE (types of events and number) and the event rate of each MACE component were presented in online supplemental table 1). In the sensitivity analysis using BMI and LVEF as the continuous variable in the multivariable Cox proportional hazard models, the trends of the primary and secondary endpoints were fully consistent with the main analysis (online supplemental table 2). In the subgroup analyses, there were no significant interactions between the subgroup factors such as ischaemic heart disease, chronic kidney disease, LAVI, and RWT and the effect of LVH groups to the no LVH on the primary outcome measure (online supplemental figure 1).

\section{Additional analyses: Cornell voltage and Sokolow-Lyon voltage as ECG criteria}

When we used the Cornell voltage as the ECG criteria for LVH (online supplemental table 3), the KaplanMeier curves were mostly consistent with the main analysis (online supplemental figure 2A). After adjusting for confounders, the risk of those with both ECG-based and echo-based LVH and only echo-based LVH remained significant for the primary outcome measure (online supplemental table 4). When we used the Sokolow-Lyon voltage as the ECG criteria for LVH (online supplemental table 5), the Kaplan-Meier curves were mostly consistent with the main analysis (online supplemental figure 2B). After adjusting for confounders, the risk of those with both ECG-based and Echo-based LVH and only Echobased LVH remained significant for the primary outcome measure (online supplemental table 6). 
Table 1 Clinical, ECG and echo characteristics

\begin{tabular}{|c|c|c|c|c|c|c|c|}
\hline & $\begin{array}{l}\text { Total } \\
(n=3212)\end{array}$ & $\begin{array}{l}\text { Both ECG- } \\
\text { based and } \\
\text { echo-based } \\
\text { LVH }(n=131)\end{array}$ & $\begin{array}{l}\text { Only echo- } \\
\text { based LVH } \\
(n=156)\end{array}$ & $\begin{array}{l}\text { Only } \\
\text { ECG-based } \\
\text { LVH }(n=409)\end{array}$ & $\begin{array}{l}\text { No LVH } \\
(n=2516)\end{array}$ & $P$ value & Total N \\
\hline \multicolumn{8}{|l|}{ Clinical characteristics } \\
\hline Age, years* & $64.5 \pm 15.9$ & $68.4 \pm 13.7$ & $70.6 \pm 13.1$ & $66.9 \pm 14.2$ & $63.6 \pm 16.3$ & $<0.001$ & 3212 \\
\hline$>70$ years & $1371(42.7)$ & $67(51.1)$ & $92(59.0)$ & $201(49.1)$ & $1011(40.2)$ & $<0.001$ & 3212 \\
\hline Women* & $1512(47.0)$ & $77(58.8)$ & $81(51.9)$ & $201(49.1)$ & $1153(45.8)$ & 0.01 & 3212 \\
\hline $\mathrm{BMI} \mathrm{kg/ \textrm {m } ^ { 2 }}$ & $23.2 \pm 4.2$ & $23.6 \pm 4.8$ & $23.8 \pm 5.2$ & $23.5 \pm 4.5$ & $23.1 \pm 4.0$ & 0.054 & 3202 \\
\hline$>25 \mathrm{~kg} / \mathrm{m}^{2 *}$ & 915 (28.6) & $42(32.1)$ & $58(37.2)$ & $135(33.1)$ & $680(27.1)$ & 0.004 & 3202 \\
\hline Diabetes* & $983(30.6)$ & $54(41.2)$ & $67(42.9)$ & $147(35.9)$ & $715(28.4)$ & $<0.001$ & 3212 \\
\hline Hypertension* & $1771(55.1)$ & $115(87.8)$ & $119(76.3)$ & $257(62.8)$ & $1280(50.9)$ & $<0.001$ & 3212 \\
\hline Dyslipidaemia* & $941(29.3)$ & $58(44.3)$ & $60(38.5)$ & $138(33.7)$ & $685(27.2)$ & $<0.001$ & 3212 \\
\hline $\begin{array}{l}\text { Ischaemic heart } \\
\text { disease* }\end{array}$ & 979 (30.5) & $63(48.1)$ & $64(41.0)$ & $125(30.6)$ & 727 (28.9) & $<0.001$ & 3212 \\
\hline $\begin{array}{l}\text { Chronic kidney } \\
\text { disease* }^{*}\end{array}$ & $449(14.0)$ & $49(37.4)$ & $51(32.7)$ & $64(15.7)$ & $285(11.3)$ & $<0.001$ & 3212 \\
\hline Atrial fibrillation* & $267(8.3)$ & $15(11.5)$ & $14(9.0)$ & $38(9.3)$ & $200(7.9)$ & 0.43 & 3212 \\
\hline \multicolumn{8}{|l|}{ ECG characteristics } \\
\hline Heart rate, bpm & $71.6 \pm 15.6$ & $73.8 \pm 15.7$ & $70.9 \pm 16.3$ & $72.5 \pm 17.9$ & $71.4 \pm 15.1$ & 0.18 & 3212 \\
\hline QRS duration & $96.6 \pm 9.0$ & $104.3 \pm 8.3$ & $96.8 \pm 9.0$ & $102.2 \pm 8.2$ & $95.2 \pm 8.6$ & $<0.001$ & 3212 \\
\hline $\begin{array}{l}\text { Sokolow-Lyon } \\
\text { voltage LVH }\end{array}$ & $472(14.7)$ & $57(43.5)$ & $48(30.8)$ & $114(27.9)$ & $253(10.1)$ & $<0.001$ & \\
\hline $\begin{array}{l}\text { Cornell voltage, } \\
\mathrm{mm}\end{array}$ & $15.8 \pm 7.1$ & $28.1 \pm 8.0$ & $15.5 \pm 4.7$ & $25.6 \pm 5.3$ & $13.6 \pm 5.1$ & $<0.001$ & 3212 \\
\hline Cornell voltage LVH & 367 (11.4) & $95(72.5)$ & $6(3.8)$ & $237(57.9)$ & $29(1.2)$ & $<0.001$ & \\
\hline $\begin{array}{l}\text { Cornell product, } \\
\mathrm{mm} \cdot \mathrm{ms}\end{array}$ & $1811.9 \pm 744.6$ & $3297.9 \pm 843.7$ & $1793.1 \pm 449.5$ & $2917.6 \pm 516.7$ & $1556.0 \pm 485.7$ & $<0.001$ & 3212 \\
\hline \multicolumn{8}{|l|}{ Echo characteristics } \\
\hline LVDd, cm & $4.65 \pm 0.55$ & $5.22 \pm 0.63$ & $5.23 \pm 0.67$ & $4.67 \pm 0.52$ & $4.58 \pm 0.50$ & $<0.001$ & 3212 \\
\hline LVDs, cm & $3.10 \pm 0.50$ & $3.70 \pm 0.77$ & $3.61 \pm 0.71$ & $3.12 \pm 0.49$ & $3.04 \pm 0.42$ & $<0.001$ & 3212 \\
\hline IVSTd, cm & $0.81 \pm 0.15$ & $1.01 \pm 0.18$ & $0.99 \pm 0.16$ & $0.83 \pm 0.14$ & $0.78 \pm 0.13$ & $<0.001$ & 3212 \\
\hline LVPWd, cm & $0.79 \pm 0.14$ & $0.98 \pm 0.18$ & $0.96 \pm 0.15$ & $0.82 \pm 0.13$ & $0.77 \pm 0.12$ & $<0.001$ & 3212 \\
\hline RWT & $0.34 \pm 0.07$ & $0.38 \pm 0.09$ & $0.38 \pm 0.09$ & $0.36 \pm 0.07$ & $0.34 \pm 0.06$ & $<0.001$ & 3212 \\
\hline$>0.42$ & $370(11.5)$ & 39 (29.8) & $38(24.4)$ & $62(15.2)$ & $231(9.2)$ & $<0.001$ & 3212 \\
\hline LVMI, $g / m^{2}$ & $75.4 \pm 21.7$ & $122.7 \pm 23.6$ & $118.7 \pm 17.2$ & $78.9 \pm 15.8$ & $69.7 \pm 15.7$ & $<0.001$ & 3212 \\
\hline $\mathrm{LAD}, \mathrm{cm}$ & $3.48 \pm 0.64$ & $3.96 \pm 0.62$ & $3.98 \pm 0.74$ & $3.59 \pm 0.66$ & $3.41 \pm 0.61$ & $<0.001$ & 3210 \\
\hline LAVI, $\mathrm{mL} / \mathrm{m}^{2}$ & $22.2 \pm 10.6$ & $32.4 \pm 13.6$ & $32.3 \pm 17.2$ & $24.0 \pm 11.1$ & $20.8 \pm 9.1$ & $<0.001$ & 2901 \\
\hline$>34 \mathrm{~mL} / \mathrm{m}^{2}$ & $293(10.1)$ & 39 (33.3) & $46(33.8)$ & 47 (12.9) & $161(7.1)$ & $<0.001$ & 2901 \\
\hline $\mathrm{EF}, \%$ & $61.9 \pm 7.0$ & $55.7 \pm 12.1$ & $57.5 \pm 10.9$ & $61.8 \pm 7.7$ & $62.5 \pm 5.8$ & $<0.001$ & 3212 \\
\hline$<50 \%$ \% & $185(5.8)$ & 33 (25.2) & 31 (19.9) & 27 (6.6) & $94(3.7)$ & $<0.001$ & 3212 \\
\hline
\end{tabular}

Comparisons among four groups were performed using the $\chi^{2}$ test for categorical variables, and one-way analysis of variance for continuous variables. Values are number (\%), mean \pm SD

ECG criteria for LVH was defined as the Cornell product $>2440 \mathrm{~mm} \cdot \mathrm{ms}$.

${ }^{*}$ Potential risk-adjusting variables selected for cox proportional hazard model.

$\mathrm{BMI}$, body mass index; EF, ejection fraction; HR, heart rate; IVSTd, diastolic interventricular septal wall thickness; LAVI, left atrial volume index; LVDd, left ventricular diastolic dimension; LVDs, left ventricular systolic dimension; LVH, left ventricular hypertrophy; LVMI, left ventricular mass index; LVPWd, diastolic left ventricular posterior wall thickness; ; RWT, relative wall thickness. 
A

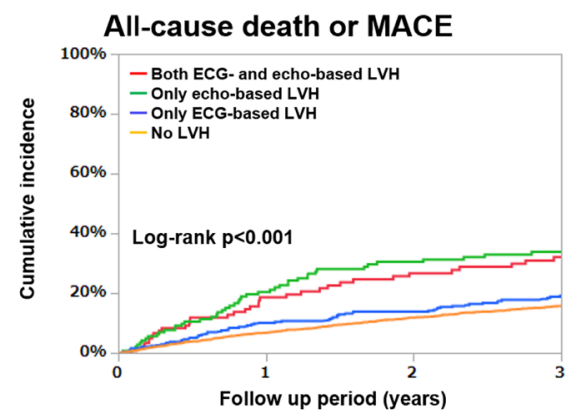

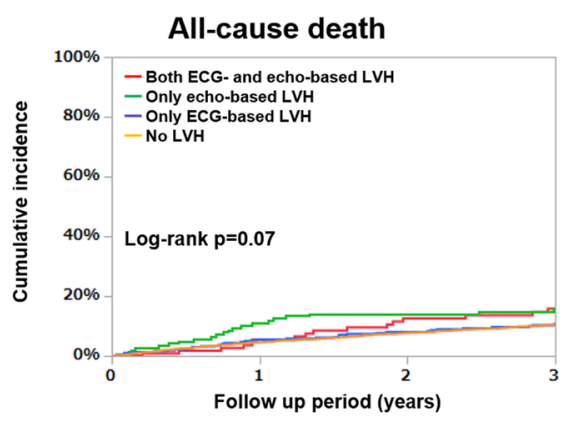

C

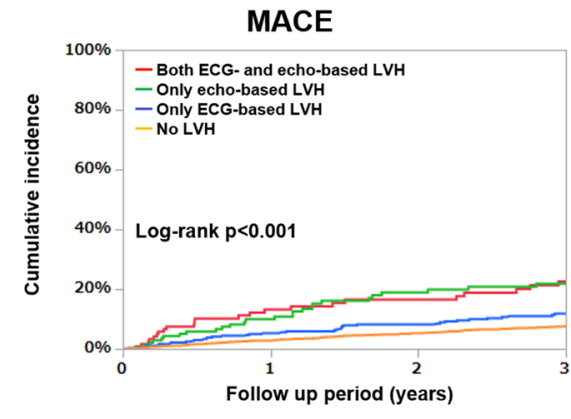

\begin{tabular}{|c|c|c|c|c|c|c|c|c|c|c|c|c|c|c|}
\hline Interval (Years) & $\mathbf{0}$ & 1 & 2 & 3 & Interval (Years) & 0 & 1 & 2 & 3 & Interval (Years) & 0 & 1 & 2 & 3 \\
\hline Both ECG- and echo-based LVH & & & & & Both ECG- and echo-bas & & & & & Both ECG- and echo-based LVH & & & & \\
\hline $\mathrm{N}$ of patients with at least 1 event & & 21 & 29 & 34 & $\mathrm{~N}$ of patients with event & & 6 & 13 & 16 & $\mathrm{~N}$ of patients with at least 1 event & & 15 & 18 & 23 \\
\hline $\mathrm{N}$ of patients at risk & 131 & 82 & 70 & 62 & $\mathrm{~N}$ of patients at risk & 131 & 97 & 84 & 76 & $\mathrm{~N}$ of patients at risk & 131 & 82 & 70 & 62 \\
\hline Cumulative incidence & & $18.6 \%$ & $26.7 \%$ & $32.0 \%$ & Cumulative incidence & & $5.5 \%$ & $12.6 \%$ & $15.8 \%$ & Cumulative incidence & & $13.2 \%$ & $16.5 \%$ & $22.5 \%$ \\
\hline Only echo-based LVH & & & & & Only echo-based LVH & & & & & Only echo-based LVH & & & & \\
\hline $\mathrm{N}$ of patients with at least 1 event & & 28 & 41 & 45 & $\mathrm{~N}$ of patients with event & & 15 & 19 & 21 & $\mathrm{~N}$ of patients with at least 1 event & & 13 & 23 & 26 \\
\hline $\mathrm{N}$ of patients at risk & 156 & 105 & 86 & 74 & $\mathrm{~N}$ of patients at risk & 156 & 115 & 103 & 89 & $\mathrm{~N}$ of patients at risk & 156 & 105 & 86 & 74 \\
\hline Cumulative incidence & & $20.4 \%$ & $30.4 \%$ & $33.8 \%$ & Cumulative incidence & & $11.0 \%$ & $14.1 \%$ & $15.9 \%$ & Cumulative incidence & & $9.9 \%$ & $18.9 \%$ & $21.8 \%$ \\
\hline Only ECG-based LVH & & & & & Only ECG-based LVH & & & & & Only ECG-based LVH & & & & \\
\hline $\mathrm{N}$ of patients with at least 1 event & & 37 & 49 & 65 & $\mathrm{~N}$ of patients with event & & 20 & 28 & 36 & $\mathrm{~N}$ of patients with at least 1 event & & 19 & 28 & 38 \\
\hline $\mathrm{N}$ of patients at risk & 409 & 307 & 273 & 222 & $\mathrm{~N}$ of patients at risk & 409 & 322 & 291 & 245 & $\mathrm{~N}$ of patients at risk & 409 & 307 & 273 & 222 \\
\hline Cumulative incidence & & $10.1 \%$ & $13.7 \%$ & $19.2 \%$ & Cumulative incidence & & $5.5 \%$ & $7.9 \%$ & $10.7 \%$ & Cumulative incidence & & $5.3 \%$ & $8.2 \%$ & $11.8 \%$ \\
\hline No LVH & & & & & No LVH & & & & & No LVH & & & & \\
\hline $\mathrm{N}$ of patients with at least 1 event & & 144 & 240 & 307 & $\mathrm{~N}$ of patients with event & & 96 & 157 & 203 & $N$ of patients with at least 1 event & & 59 & 103 & 139 \\
\hline $\mathrm{N}$ of patients at risk & 2516 & 1872 & 1586 & 1362 & $\mathrm{~N}$ of patients at risk & 2516 & 1915 & 1659 & 1447 & $\mathrm{~N}$ of patients at risk & 2516 & 1872 & 1586 & 1362 \\
\hline Cumulative incidence & & $6.7 \%$ & $11.8 \%$ & $15.7 \%$ & Cumulative incidence & & $4.5 \%$ & $7.7 \%$ & $10.4 \%$ & Cumulative incidence & & $2.8 \%$ & $5.2 \%$ & $7.5 \%$ \\
\hline
\end{tabular}

Figure 2 Cumulative incidence of the primary outcome measure (a composite of all-cause death or MACE) and secondary outcomes measure (all cause death, MACE). (A) a composite of all-cause death or MACE, (B) all-cause death, (C) MACE. LVH, left ventricular hypertrophy; MACE, major adverse cardiovascular event.

\section{DISCUSSION}

The results of this study illustrated that (1) a substantial proportion of patients diagnosed with no LVH based on ECG criteria had an echo-based LVH, and those with only echo-based LVH were older and more likely to have chronic kidney disease and a higher LAVI, (2) the outcomes in patients with only echo-based LVH, as well as those with both ECG-based and echo-based LVH, were worse than those in patients without $\mathrm{LVH}$ and 3) the risk in patients with ECG-based LVH without echo-based LVH was numerically high; however, after adjustment, the risk became insignificant.

Due to the larger sample size than that in a previous report, ${ }^{6}$ we can show the stepwise increase in cumulative incidence among ECG-based and/or echo-based LVH. Echo-based LVH is an established marker for cardiovascular events in the general population and in patients with cardiovascular diseases. ${ }^{3}{ }^{4}$ Although ECG-based LVH criteria have limited sensitivity for detecting actual myocardial hypertrophy, ${ }^{9}$ these markers based on QRS amplitude have good specificity for physiological LVH. In fact, previous studies have shown that ECG-based LVH is a strong predictor of cardiovascular mortality and morbidity. ${ }^{2}{ }^{20}$ However, it has been shown that the anatomy of the left ventricle in LVH is not the only determinant of QRS amplitude, the key feature on which almost all ECG-LVH criteria depend on. ${ }^{21}{ }^{22}$ Slowed conduction due to changes in the sequence of ventricular activation, such as fibrosis, could lead to ECG-based LVH even in situations where the anatomy of the left ventricle is not changed. When we evaluated ECG-based LVH and echo-based LVH simultaneously, the event rate was highest in patients with only echo-based LVH, followed by those with both ECG- and echo-based LVH, those with only ECG-based LVH and those without ECG-based or echo-based LVH.

Recent studies reported that MRI improves the diagnostic accuracy of ECG-LVH. ${ }^{23}{ }^{24}$ Bacharova et al reported a discrepancy between LVH on MRI and electrocardiographic hypertrophy. ${ }^{25}$ They reported that both ECGbased and MRI-based LVH and only MRI-based LVH showed a strong association with incident CVD events compared with patients without LVH. Our results were consistent with those of previous studies in which LVH found only by imaging was associated with worse clinical outcomes. Structural and functional information of the heart can be detected more easily using echo than by MRI. Further studies are needed to determine the difference in the accuracy of detecting LVH between echo and MRI.

In the present study, the prognosis of patients who had echo-based LVH but not ECG-based LVH was clearly highlighted. There are several possible reasons for the poor outcomes in these patients. First, the loss of healthy myocardium is a known cause of low QRS voltage. Myocardial ischemia, increased myocardial fibrosis and 


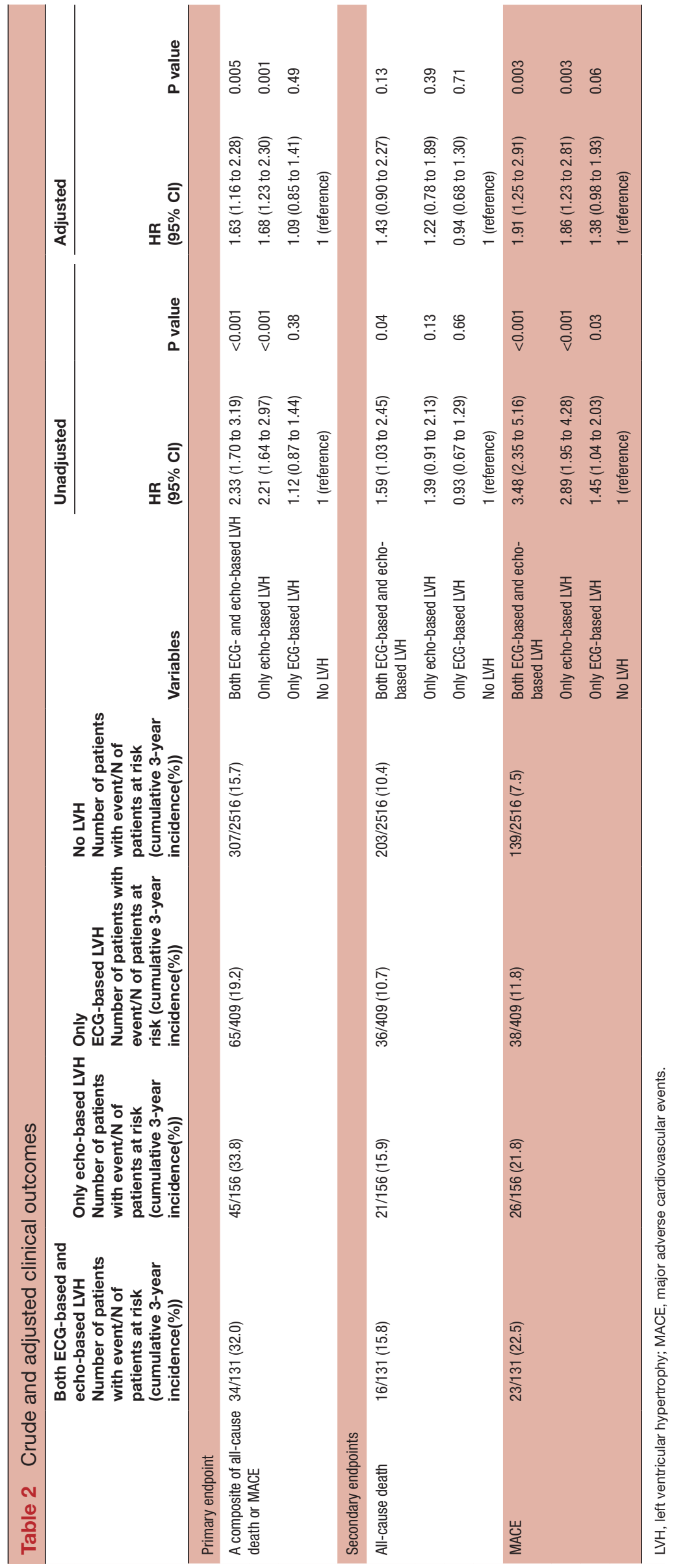


the accumulation of amyloid protein, all of which are accompanied by advanced age and restrictive physiology, are suggested mechanisms of the relatively low voltage of ECG, which indicates myocardial damage. ${ }^{26-28}$ Patients with only echo-based LVH were characterised as having advanced age with almost normal LVEF. Although the detailed pathophysiology could not be assessed due to the retrospective nature of the present study, the abovementioned involvement of ischemia, fibrosis and cardiac amyloidosis should be considered as a differential diagnosis. Second, increased electrical resistance due to airfilled bullae is also one of the contributors to reduced QRS voltage in emphysema. ${ }^{2930}$ The main cause of emphysema is smoking, which is also a risk factor for cardiovascular diseases. Altogether, the outcomes of patients with only echo-based LVH were worse than those with both ECG-based and echo-based LVH.

The prognostic value of ECG-based LVH, but not echo-based LVH, was neutral in the present study. ECG provides unique information on the electric field of the heart and not the information of the LV size/dimensions. Patients with ECG-based LVH, but not echo-based LVH, had similar echocardiographic and clinical characteristics to patients in the no LVH group. These patients had less cardiac damage, such as cardiac chamber enlargement and cardiac dysfunction, and fewer comorbidities than those with echo-based LVH. Consequently, patients with ECG-based LVH alone were not associated with the 3-year worse outcomes. However, whether ECG-based LVH and non-LVH groups have comparable long-term prognoses remains to be elucidated.

The ratio of patients who had ECG-based LVH but not echo-based LVH was higher than that in a previous study. ${ }^{25}$ This is likely related to the habitus of the Japanese population. Other possible reasons for the result include different backgrounds such as racial difference and the fact that the population of our study includes various situation's echocardiography such as in outpatients, preoperative patients and inpatients. When we used ECG to detect echo-based LVH as a gold standard, the sensitivity and specificity varied among the calculation methods. ${ }^{2031} 32$ To generalise these findings, more prospective studies in the general population or in studies that search for a more targeted population are warranted. In clinical settings, when we see the mismatch between ECG-based LVH and echo-based LVH, the underlying mechanism that causes the mismatch would be investigated thoroughly in order to understand and treat the pathophysiologic condition in each patient.

\section{Limitations}

This study has several limitations. First, ECG and transthoracic echocardiograms were ordered at the discretion of the treating physician, with no standardised indications. ${ }^{78}$ Second, patient data were extracted from their electronic medical records, which resulted in a low follow-up rate, especially at 3 years. In addition, information on the symptoms was not included. Thus, we had no data regarding the proportion of patients with symptomatic heart failure. Third, this was a single-centre study performed in Japan; thus, selection bias cannot be excluded despite the large sample size. Fourth, we did not have the data about the presence of a strain pattern at the ECG. Finally, unmeasured confounders affecting prognosis remain.

\section{CONCLUSIONS}

Echo-based LVH without ECG-based LVH was associated with a higher risk of adverse clinical outcomes, and the risk was comparable to that in patients with both echobased and ECG-based LVH in a hospital-based patient in Japan.

Contributors YS and TK: conceived the design, performed statistical analysis and wrote manuscript. YY, YH, EN, TH and Ml: collected the data and made critical revision.

Funding The authors have not declared a specific grant for this research from any funding agency in the public, commercial or not-for-profit sectors.

Competing interests None declared.

Patient consent for publication Not applicable.

Ethics approval The institutional review board of Kitano Hospital (approval number P16-02-005).

Provenance and peer review Not commissioned; externally peer reviewed.

Data availability statement Data are available upon reasonable request. All relevant data are within the manuscript. The raw data will be provided upon the reasonable request to the corresponding author.

Open access This is an open access article distributed in accordance with the Creative Commons Attribution Non Commercial (CC BY-NC 4.0) license, which permits others to distribute, remix, adapt, build upon this work non-commercially, and license their derivative works on different terms, provided the original work is properly cited, appropriate credit is given, any changes made indicated, and the use is non-commercial. See: http://creativecommons.org/licenses/by-nc/4.0/.

ORCID iD

Takao Kato http://orcid.org/0000-0001-8213-7999

\section{REFERENCES}

1 Rautaharju PM, Soliman EZ. Electrocardiographic left ventricular hypertrophy and the risk of adverse cardiovascular events: a critical appraisal. J Electrocardiol 2014;47:649-54.

2 Hsieh BP, Pham MX, Froelicher VF. Prognostic value of electrocardiographic criteria for left ventricular hypertrophy. Am Heart J 2005;150:161-7.

3 Bluemke DA, Kronmal RA, Lima JAC, et al. The relationship of left ventricular mass and geometry to incident cardiovascular events: the MESA (multi-ethnic study of atherosclerosis) study. J Am Coll Cardiol 2008;52:2148-55.

4 de Simone G, Gottdiener JS, Chinali M, et al. Left ventricular mass predicts heart failure not related to previous myocardial infarction: the cardiovascular health study. Eur Heart J 2008;29:741-7.

5 Maanja M, Wieslander B, Schlegel TT, et al. Diffuse myocardial fibrosis reduces electrocardiographic voltage measures of left ventricular hypertrophy independent of left ventricular mass. J Am Heart Assoc 2017;6:e003795.

6 Sundström J, Lind L, Arnlöv J, et al. Echocardiographic and electrocardiographic diagnoses of left ventricular hypertrophy predict mortality independently of each other in a population of elderly men. Circulation 2001;103:2346-51.

7 Seko Y, Kato T, Haruna T, et al. Association between atrial fibrillation, atrial enlargement, and left ventricular geometric remodeling. Sci Rep 2018;8:6366.

8 Seko Y, Kato T, Morita Y, et al. Impact of left ventricular concentricity on long-term mortality in a hospital-based population in Japan. PLoS One 2018;13:e0203227. 
9 Hancock EW, Deal BJ, Mirvis DM, et al. AHA/ACCF/HRS recommendations for the standardization and interpretation of the electrocardiogram: Part V: electrocardiogram changes associated with cardiac chamber hypertrophy: a scientific statement from the American Heart Association Electrocardiography and Arrhythmias Committee, Council on Clinical Cardiology; the American College of Cardiology Foundation; and the Heart Rhythm Society: endorsed by the International Society for Computerized Electrocardiology. Circulation 2009;119:e251-61.

10 Okin PM, Wachtell K, Gerdts E, et al. Relationship of left ventricular systolic function to persistence or development of electrocardiographic left ventricular hypertrophy in hypertensive patients: implications for the development of new heart failure. $J$ Hypertens 2014;32:2472-8. discussion 2478.

11 Okin PM, Wachtell K, Devereux RB, et al. Regression of electrocardiographic left ventricular hypertrophy and decreased incidence of new-onset atrial fibrillation in patients with hypertension. JAMA 2006;296:1242-8.

12 Molloy TJ, Okin PM, Devereux RB, et al. Electrocardiographic detection of left ventricular hypertrophy by the simple QRS voltageduration product. J Am Coll Cardiol 1992;20:1180-6.

13 Okin PM, Roman MJ, Devereux RB, et al. Electrocardiographic identification of increased left ventricular mass by simple voltageduration products. J Am Coll Cardiol 1995;25:417-23.

14 Seko Y, Kato T, Morita Y, et al. Age- and body size-adjusted left ventricular end-diastolic dimension in a Japanese hospital-based population. Circ J 2019;83:604-13

15 Seko Y, Kato T, Morita Y, et al. Association with left atrial volume index and long-term prognosis in patients without systolic dysfunction nor atrial fibrillation: an observational study. Heart Vessels 2020;35:223-31.

16 Seko Y, Kato T, Shiba M, et al. Staging cardiac damage in patients with hypertension. Hypertension 2019;74:1357-65.

17 Seko Y, Kato T, Shiba M, et al. Association of the low E' and high E/e' with long-term outcomes in patients with normal ejection fraction: a hospital population-based observational cohort study. BMJ Open 2019;9:e032663.

18 Seko Y, Kato T, Shiba M, et al. Isolated tricuspid regurgitation and long-term outcome in patients with preserved ejection fraction. Circ Rep 2019;1:617-22.

19 Lang RM, Badano LP, Mor-Avi V, et al. Recommendations for cardiac chamber quantification by echocardiography in adults: an update from the American Society of echocardiography and the European association of cardiovascular imaging. J Am Soc Echocardiogr 2015;28:1-39.
20 Ishikawa J, Ishikawa S, Kario K, et al. Levels of Cornell voltage and Cornell product for predicting cardiovascular and stroke mortality and morbidity in the general Japanese population. Circ $J$ 2014;78:465-75.

21 Bacharova L, Szathmary V, Mateasik A. QRS complex and ST segment manifestations of ventricular ischemia: the effect of regional slowing of ventricular activation. J Electrocardiol 2013;46:497-504.

22 Bacharova L, Szathmary V, Kovalcik M, et al. Effect of changes in left ventricular anatomy and conduction velocity on the QRS voltage and morphology in left ventricular hypertrophy: a model study. $J$ Electrocardiol 2010;43:200-8.

23 Sparapani R, Dabbouseh NM, Gutterman D, et al. Detection of left ventricular hypertrophy using Bayesian additive regression trees: the MESA. J Am Heart Assoc 2019;8:e009959.

24 Jain A, Tandri H, Dalal D, et al. Diagnostic and prognostic utility of electrocardiography for left ventricular hypertrophy defined by magnetic resonance imaging in relationship to ethnicity: the multiethnic study of atherosclerosis (MESA). Am Heart J 2010;159:652-8.

25 Bacharova L, Chen $\mathrm{H}$, Estes EH, et al. Determinants of discrepancies in detection and comparison of the prognostic significance of left ventricular hypertrophy by electrocardiogram and cardiac magnetic resonance imaging. Am J Cardiol 2015;115:515-22.

26 Bär FW, Brugada P, Dassen WR, et al. Prognostic value of $Q$ waves, R/S ratio, loss of $R$ wave voltage, ST-T segment abnormalities, electrical axis, low voltage and notching: correlation of electrocardiogram and left ventriculogram. J Am Coll Cardiol 1984;4:17-27.

27 Madias JE. Low QRS voltage and its causes. J Electrocardiol 2008;41:498-500.

28 Kampaktsis PN, Ullal AV, Swaminathan RV, et al. Absence of electrocardiographic left ventricular hypertrophy is associated with increased mortality after transcatheter aortic valve replacement. Clin Cardiol 2018;41:1246-51.

29 Rodman DM, Lowenstein SR, Rodman T. The electrocardiogram in chronic obstructive pulmonary disease. J Emerg Med 1990;8:607-15.

30 Larssen MS, Steine K, Hilde JM, et al. Mechanisms of ECG signs in chronic obstructive pulmonary disease. Open Heart 2017;4:e000552.

31 Shirai T, Kasao M, Nozaki M, et al. Evaluation of hypertensive cardiac abnormalities using the Cornell product. Circ J 2007;71:731-5.

32 Su F-Y, Li Y-H, Lin Y-P, et al. A comparison of Cornell and SokolowLyon electrocardiographic criteria for left ventricular hypertrophy in a military male population in Taiwan: the cardiorespiratory fitness and hospitalization events in armed forces study. Cardiovasc Diagn Ther 2017;7:244-51. 